\title{
Trois défis inhérents à une démarche thématique intégrée d'enseignement- apprentissage médiatisé du français langue seconde à l'université
}

\author{
Linda de Serres \\ Université du Québec à Trois-Rivières, CANADA \\ linda.de.serres@uqtr.ca
}

Compte rendu d'expérience

\section{Résumé}

Malgré sa pléthore de ressources, Internet en soi ne constitue pas un outil d'avant-plan dans l'enseignement-apprentissage du français langue seconde (FLS) à l'université. S'appuyant sur cette prémisse, nous avons pour ambition d'exposer d'abord dans cet article les failles d'Internet, puis de montrer en quoi il peut, en revanche, dans une démarche thématique médiatisée et intégrée, constituer une véritable plus-value en FLS. Au fil d'une exemplification en ce sens sur le thème du reportage radiophonique, nous aborderons l'ampleur des défis rencontrés : le temps nécessaire à la conception du matériel, l'anxiété envers les technologies, le nouveau rôle du professeur. De-ci de-là, des avenues pour mieux tirer profit des ressources Internet dans la classe de FLS à l'université seront proposées.

\begin{abstract}
Though it provides a dizzying number of resources, Internet does not constitute a primary tool in the teaching and learning of French as a second language (FSL) at the university level. Taking this as our initial premise, this paper will first address some of the weaknesses of Internet in this matter, and then show, conversely, how Internet can be very helpful if duly integrated into a pedagogical, thematic project. Through the example of a radio report scenario, this paper will examine three challenges facing teachers: time required for design, technology anxiety, and the teacher's role. Ways of enhancing the use of Internet resources in the FSL classroom will be given.
\end{abstract}

(C)Auteur(s). Cette œuvre, disponible à http://ritpu.ca/IMG/pdf/ritpu_0203_deserres.pdf, est mise à disposition selon les termes de la licence Creative Commons Attribution - Pas de Modification 2.5 Canada : http://creativecommons.org/licences/by-nd/2.5/ca/deed.fr 


\section{Lacunes d'Internet}

Bien que les sites de français langue seconde (FLS) foisonnent dans Internet, l'apprenant laissé à lui-même ne parvient quà en retirer des informations brutes et parcellaires. Quand un professeur lui suggère de pratiquer une notion linguistique donnée à partir de différents sites triés sur le volet (de Serres, 2003, 2004c; Lebeaupin, 1998), l'apprenant n'éprouve qu'une satisfaction mitigée, cause d'une "acquisition de connaissances morcelées, isolées" (Tardif, 2000, p.120), une "véritable balkanisation des informations" (Lebrun, 2002, p. 149). Internet offre un grand nombre d'exercices de répétitions et de transformation en FLS. Malheureusement, plusieurs ne consistent qu'en des exercices spécifiques et redondants (à titre d'illustration, voir Perrot (n.d.) ou Azurlingua, n.d.). Pire encore, tel qu'en témoignent Germain et Netten (2004), les activités dites "interactives", avec des phrases à réécrire ou des exercices à trous, ne s'inscrivent pas dans un "modèle d'apprentissage actualisé » en faveur d'une autonomie langagière en FLS (pour des exemples, voir Exercices interactifs, n.d.): " $[\mathrm{De}]$ nombreux "exercices à trous" ou "blancs à compléter" à l'ordinateur, [...] [sont] très souvent proposés aux élèves. [...] Le modèle d'apprentissage sous-jacent nous paraît être de nature plutôt béhavioriste que constructiviste ou socio-constructiviste (p. 10).

Internet en tant qu'outil technologique consiste en une avenue pédagogique parmi d'autres à adopter dans l'enseignement-apprentissage d'une langue: «Technology-based language teaching is not a method but is integrated into various pedagogical approaches" (Kern, 2006, p. 200). Internet n'induit pas les interactions entre pairs; il revient au pédagogue de les provoquer. Et dans un contexte d'enseignement-apprentissage d'une LS, cela s'impose d'autant plus puisque l'apprenant qui acquiert une langue est, à tout le moins sur le plan linguistique, peu outillé pour poser des choix éclairés quant à la qualité des contenus de sites donnés (Crystal, 2001) et à la pertinence du type d'activités qui y est proposé. Comme le souligne Kern (2006), c'est la façon dont on recourt avant tout à une technologie qui influence l'apprentissage d'une langue et d'une culture. En ce sens, la pédagogie et le pédagogue même représentent des éléments clés.

"There is a consensus in CALL research [computer-assisted language learning] that it is not technology per se that affects the learning of language and culture but the particular uses of technology. This emphasis on use highlights the central importance of pedagogy and the teacher (Kern, 2006, p. 200).
Une question s'impose alors : comment le pédagogue peut-il mettre à profit les potentialités d'Internet pour marquer un " progrès pédagogique " conduisant à l'interaction dans la classe de langue? Internet, pourtant intarissable, engendre chez l'apprenant de FLS soit un sentiment de désorientation (de Serres, 2004b), soit un sentiment de lassitude et d'insatisfaction. Comment, dans pareil cas, parvenir à créer un climat agréable et enrichissant tant pour l'apprenant que pour le professeur? En un mot, quel outil permettrait de tirer le meilleur parti des caractéristiques intrinsèques d'Internet dans un souci de bonifier l'enseignement-apprentissage de la langue française? Tel est le fondement de ce "macrodéfi ", un défi ambitieux, à relever impérativement: concevoir une ressource pédagogique souple, laquelle, paradoxalement, s'insérerait dans un cadre de travail circonscrit.

Dans les prochaines pages, nous proposerons une façon parmi d'autres de pallier les lacunes d'Internet dans l'enseignement-apprentissage d'une LS. Cette démonstration cédera la place ici et là aux trois principaux défis inhérents à cette démarche. Il s'agit d'une démarche qui sous-tend la création de scénarios pédagogiques médiatisés.

\section{Le scénario pédagogique médiatisé : le définir, le concevoir}

Le scénario pédagogique médiatisé consiste en une suite pédagogisée qui met à profit des contenus de sites offerts dans Internet. Un seul exemple suffira à illustrer nos propos : celui d'un scénario axé sur le reportage radiophonique. Conçue à l'intention d'apprenants universitaires de niveau avancé en FLS, la suite agencée de consultations en ligne, qui souscrit à une préoccupation pédagogique sur le sujet que représente la radio, comporte quatre séances de travail d'environ 60 minutes chacune. Cette durée arbitraire a été retenue pour faciliter le travail des professeurs qui doivent réserver des périodes pour avoir accès à une salle multimédia. Chaque séance d'une heure peut comporter plus d'une activité distincte à exécuter dans l'ordre et toutes liées à la thématique (p. ex.: A.1, A.2, A.3, A.4). Le changement alpha signale une autre séance de 60 minutes; le repère ordinal quant à lui s'affiche en continu (p. ex.: B.5; C.6, C.7; D.8). Une telle façon de faire facilite les repères lors du travail au sein du groupe (entre pairs, entre le professeur et les étudiants) lorsque, par exemple, des ajustements sont requis. À partir de quelque 20 sites s'atomisent au sein du scénario en question, tous types confondus, neuf objectifs :

1. Découvrir et apprécier l'univers de la radio

2. Prendre conscience de la recherche, de la réflexion et du travail d'écriture quexige la réalisation d'un reportage radiophonique 
3. Effectuer une recherche en autonomie dans Internet

4. Mettre en œuvre sa pensée créatrice

5. Structurer sa pensée

6. Développer l'expression orale

7. Développer une sensibilité face à son environnement

8. Travailler en équipe, coopérer

9. Développer son sens des responsabilités.

Le choix d'activités ou de documents prélevés dans Internet, intégrés à un scénario, «assure [...] un cadre pédagogique et permet $[. .$.$] à tout utilisateur éventuel de bien situer l'activité$ dans un contexte pédagogique " (Isabelle, 2002, p. 142). Dans l'exemple qui nous préoccupe, un paragraphe d'introduction oriente tant le pédagogue que l'apprenant quant au contexte du travail à entreprendre.

«Le téléphone, le télécopieur, l'Internet, la télévision, le cinéma et la radio sont des moyens de communication mis à notre disposition. Ce scénario vous permettra de vous pencher plus avant sur l'un d'entre eux : la radio. Devenez, sans peine aucune, reporter d'un jour! Il y a beaucoup à apprendre sur ce médium aujourd'hui noyé parmi tant d'autres, occupant toutefois, jadis, une place de premier plan... Voyez et entendez par vous-même! »

Concevoir une seule séance de travail d'une durée de $60 \mathrm{mi}$ nutes d'un scénario peut nécessiter jusquà 30 heures de préparation (Rézeau, 2001). Il s'agit du premier défi de taille: la démarche de conception. Une démarche qui requiert du temps, beaucoup de temps. Le temps investi couvre la sélection de sites, l'agencement de leurs contenus à des activités données propres à un apprentissage signifiant du FLS en lien avec un choix de thèmes variés, et, pour peaufiner les ébauches de scénarios, des essais pilotes en salle multimédia de concert avec des pédagogues et des apprenants. ${ }^{1}$ À lui seul, le souci de rendre un apprentissage signifiant soustend des principes: un "ancrage sur des connaissances antérieures, une forte contextualisation initiale, une structuration hiérarchique, une gestion métacognitive " (Tardif, 2000, p. 43). Par exemple, dans le scénario ici abordé, une grille sondage que nous avons adaptée, intitulée la radio et moi (étape A.1), invite d'entrée de jeu le principal intéressé à l'introspection. Il nous semble important dès le départ de faire réfléchir l'apprenant sur le sujet. Il s'y adonnera seul d'abord, puis avec un pair. Quelques questions auxquelles doivent répondre les apprenants les amènent à se pencher sur la place qu'occupe la radio dans leur quotidien et sur ce que ce média transmet.

\section{A.1 La grille sondage : la radio et moi}

Durée : \pm 10 minutes

1. Quelle place accordez-vous à la radio?

2. En dyade, répondez à l'oral aux questions suivantes² :

a. Quelle est l'utilité ou la fonction de la radio?

b. La radio a-t-elle une influence sur vous? Sur les gens en général? Expliquez en quoi.

c. La radio véhicule-t-elle des valeurs? Lesquelles? S'agit-il des mêmes valeurs que celles qui sont véhiculées par les autres médias comme la télévision, Internet, etc.?

d. Pensez-vous toujours connaître les intentions d'un message diffusé à la radio? Expliquez.

e. Selon vous, quels sont les métiers reliés au monde de la radio?

C'est seulement après la première séance de travail de $60 \mathrm{mi}-$ nutes, qui comprend les activités A.1, A.2, A.3 et A.4 (nous y reviendrons plus loin), que nous inviterons l'apprenant à pousser son travail plus avant sur la thématique retenue, comme l'illustre l'activité B. 5 présentée infra.

\section{B.5 La recherche}

Durée : \pm 60 minutes

3. Après avoir constitué un trio, pour amorcer une répartition du travail, lisez sur la préparation du sujet à http://www.bourgoing. com/formation/radio/08preparation.htm ${ }^{3}$

4. Effectuez une recherche préliminaire sur le sujet que vous avez retenu en consultant :

a. des moteurs de recherche pour explorer des sites traitant de votre sujet, p. ex. : http://www.google.ca

b. des encyclopédies, p. ex. : http://agora.qc.ca/mot.nsf, http:// www.thecanadianencyclopedia.com ou

c. autres :

5. Lisez abondamment à propos de votre sujet

\section{Prenez des notes}

7. Cherchez-vous des extraits sonores reliés à votre sujet? Désirezvous pousser votre recherche d'information un peu plus loin?

8. Voyez les sites suivants... Vous trouverez sans doute!

a. http://radio-canada.ca/actualite/decouverte/ Pour les mordus de science, de technologies et de découvertes de toutes sortes: nature et environnement, science et société, archives 1996 à $2004 \ldots$ allez, explorez!

b. http://radio-canada.ca/actualite/semaineverte/ Pour des sujets liés à l'agriculture, la nature, la pêche : cliquez sur page reportage... et voyez reportages et archives!

c. http://radio-canada.ca/actualite/lepicerie/ Pour les gourmands : cliquez sur archives et partez à la découverte du monde de l'alimentation!

d. http://radio-canada.ca/actualite/lafacture/ Pour ceux qui sont intéressés aux questions de consommation : voyez les reportages archivés et les capsules archivées

e. http://radio-canada.ca/actualite/5sur5/ Pour des reportages sur les affaires culturelles, l'économie, l'environnement, la justice, le monde, la politique, la santé, les sciences, la société et les sports

f. http://radio-canada.ca/radio Pour explorer les différentes émissions et écouter des reportages audio de tous genres! 
g. http://www.telequebec.tv/cultive/index_cadre_H.html Pour des sujets liés à l'alimentation et à l'agriculture, cliquez sur l'image Archives capsules et explorez les capsules et les archives capsules

h. http://www.telequebec.qc.ca/lesfrancstireurs/ Pour la consultation des archives de reportages audio

i. http://www.tv5.org Pour des reportages sur différents sujets touchant la francophonie, cliquez sur WEBTV5, en bas, à droite

j. http://www.rfi.fr Pour une pléthore de documents : écouter, à l'antenne, la langue française, etc.

9. Soyez sélectifs!

La conception du scénario pédagogique médiatisé avec ses diverses activités de difficulté croissante et la variété des tâches requises (de compréhension et de production, à l'oral et à l'écrit) qui culminent avec une création de groupe en français d'un reportage radiophonique, illustre éloquemment un souci bien réel de «[... fonder l'intégration des [...] [contenus de sites Internet] sur un cadre de référence pédagogique rigoureux, qui [prend] racine sur une logique d'apprentissage" (Tardif, 2000, p.120-121). Ainsi succèdera au sondage (voir supra l'activité A.1) une activité pour cerner le métier de reporter (voir infra l'activité A.2), puis une autre, sur le reportage (voir infra l'activité A.3) et, enfin, l'apprenant sera invité à choisir un sujet à partir duquel il concevra son propre reportage (voir infra l'activité A.4 et supra l'activité B.5).

\section{A.2 Le métier de reporter}

Durée : \pm 20 minutes

1. Seul, à partir du site compagnon, visitez le http://www. bandesportive.com/reporters/entree.html

2. Cliquez sur Radio

3. Cliquez sur Pour les enseignantes et enseignants

4. Cliquez sur Les rôles de chacun

5. Prenez connaissance des différents métiers reliés au monde de la radio

6. Attardez-vous à la rubrique Les reporters

7. Lisez le court texte

8. Prenez des notes

9. Pour en savoir plus sur le métier de reporter radio, rendez-vous à http://www.studyrama.com/article.php3?id article=1114

10. Lisez le premier paragraphe

11. Complétez les phrases suivantes:

a. Le métier de reporter nécessite une vraie motivation. Le journaliste doit être

et

b. Le travail du reporter varie selon

c. Le travail de __ est un travail minutieux qui exige des qualités de rapidité et de synthèse.

\section{A.3 Le reportage}

Durée : \pm 5 minutes

1. Allez à http://www.reseau-crem.qc.ca/projet/dos7.htm
2. Sélectionnez L'information rapportée

3. Descendez la page jusqu'à la rubrique Le reportage

4. Découvrez ce qu'est un reportage en lisant les deux paragraphes

5. Prenez des notes

\section{A.4 Le choix d'un sujet}

Durée : \pm 25 minutes

1. Avant de choisir votre propre sujet de reportage radio, informezvous quant au choix et au traitement d'un sujet en général dans un reportage radiophonique à http://www.bourgoing.com/formation/ radio/02radiophonique.htm

2. Lisez les cinq pages présentées en plaçant un $X$ sur la ligne lorsque vous terminez la lecture d'un sujet (p. ex. : X) ${ }^{4}$

a. le sujet de reportage

b. un sujet « radiophonique »

c. un sujet intéressant pour vos auditeurs

d. un angle précis

e. le traitement

3. Pour passer d'une page à l'autre, choisissez la page voulue dans le menu présenté en bleu sous le titre Le choix du sujet

4. Prenez des notes

5. Il est possible de remplacer le reportage radiophonique (oral) par un reportage journalistique (écrit).

6. Le cas échéant, voyez comment réaliser un reportage journalistique à http://www.passionreportage.com/jeprendsconseil/reportages.html

7. En triade, retenez un seul sujet dans la liste thématique suivante :

\begin{tabular}{|l|l|l|}
\hline a. l'alimentation & $\begin{array}{l}\text { c. la science } \\
\text { et les découvertes }\end{array}$ & e. la consommation \\
\hline b. la nature & d. l'agriculture & f. autre: \\
\hline
\end{tabular}

Comme quiconque le déduit à la lecture des précédents contenus, les scénarios pédagogiques médiatisés commandent beaucoup de labeur et de rigueur sur le plan conceptuel'5. En revanche, ils ne requièrent aucune compétence particulière sur le plan informati$q^{6} e^{6}$, ni de la part du professeur ni de celle de l'apprenant.

\section{Action et interaction}

Tel que décrit plus tôt, le scénario pédagogique médiatisé exige de l'apprenant un recours au français, à l'oral et à l'écrit, seul et avec des pairs: dimension incontournable puisque nous savons maintenant qu'Internet est défaillant sous ce rapport. Et ce n'est pas tout. Dans l'enseignement-apprentissage d'une langue, l'action s'impose. Un apprenant doit, pour ne donner que quelques exemples, parler, écrire, se relire, partager ses réflexions, formuler des demandes et faire valoir ses opinions auprès d'autrui. En cours de travail, dans le scénario dont il possède copie sur support papier, l'apprenant effectue des actions soit pour réfléchir, soit pour structurer ses savoirs, soit pour créer. En un mot, ce document lui sert d' "outil phare" pour bâtir et consolider ses apprentissages. 
Cet « outil phare » conçu sous une forme hybride allie la technologie et le travail traditionnel avec crayon et papier, le recours aux pairs et le recours au médiateur, naguère le professeur. Au fur et à mesure qu'il suit les consignes écrites du scénario dans son document de travail, l'apprenant est invité à prendre des notes personnelles, à répondre par écrit à des questions à choix multiple ou à des questions ouvertes, à compléter des locutions ou des phrases, à composer de courts textes, à colliger ses réflexions à la suite des discussions tenues avec un ou des pairs et à préparer une présentation orale ou écrite selon les critères d'une grille d'évaluation fournie, et ainsi de suite. Dans le présent scénario, qui a trait au reportage radiophonique, après trois séances de travail de $60 \mathrm{mi}-$ nutes chacune (voir supra les activités A.1; A.2; A.3; A.4; B.5; C.6 et $C .7$ ), l'apprenant aura été progressivement préparé à présenter à ses pairs son propre reportage lors d'une quatrième et dernière séance de travail (voir infra l'activité D.8).

\section{D.8 La présentation du reportage et l'évaluation}

Durée : \pm 15 minutes par triade, à multiplier selon le nombre de triades dans le groupe

1. Vous êtes maintenant prêt à présenter votre reportage selon la formule retenue par votre médiateur (en direct ou à l'aide d'un enregistrement)

2. Si désiré, vos pairs ou votre médiateur vous évalueront, à l'oral, à partir de la grille fournie à http://csrs.qc.ca/goeland/proj/envolee/ matieres/francais/reportage/grille oral informatif.html ou, à l'écrit, à partir de la grille d'évaluation présentée à http://csrs.qc.ca/goeland/ proj/envolee/matieres/francais/reportage/grille ecrit informatif.html

3. Une suggestion : à la fin de la présentation des reportages de chaque triade de la classe, les apprenants ainsi que le médiateur peuvent voter afin de choisir le meilleur reportage et ainsi remettre, fictivement, le prix Judith-Jasmin à la triade gagnante (voir à ce sujet la variante intitulée Le prix Judith-Jasmin).

Cette façon toute particulière, non seulement d'intégrer des exercices et une évaluation aux situations d'apprentissage, mais de surcroît de se préoccuper de "l'authenticité des situations d'apprentissage » (Tardif, 2000, p. 53) souscrit étroitement à une préoccupation de maintenir un lien de cohérence tangible entre la pédagogie et Internet. De plus, comme nous l'avons déjà évoqué, «l'alternance entre les cadrages » (Perrenoud, 2004, p. 64) jumelée à la possibilité de confronter ses «[...] points de vue stimule une activité métacognitive dont chacun tire un bénéfice $[. .$.$] »$ (Perrenoud, 2004, p. 64).

Dans son document sur papier, à l'instar d'un cahier de notes, l'apprenant cumule une multitude de renseignements en lien avec le thème traité. Aussi, en classe ou hors classe, il pourra s'y référer pour maints motifs dont revoir des éléments, étudier, compléter un projet en sous-groupe ou se préparer en vue d'une évaluation. Avec de tels scénarios pédagogiques médiatisés, Internet ne consiste plus en un instantané incomplet, au hasard des trouvailles, ou en un «surfing de surface» (Lebrun, 2002, p. 149). Bien au contraire, comme nous en émettions le vœu d'entrée de jeu, Internet est mis en valeur dans un outil pédagogique au cadre bien circonscrit et souple. Le recours à Internet s'inscrit dans un processus d'apprentissage signifiant où l'apprenant s'implique et manipule la langue sous toutes ses facettes. Alimenté par un souci "d'autonomiser" l'apprenant, le scénario comporte même le corrigé des exercices ${ }^{7}$ dont nous sommes toutefois forcée de sacrifier ici la présentation par manque d'espace.

Le scénario pédagogique médiatisé constitue un concept très formateur, très complet et, au surplus, simple d'utilisation. Dans une salle multimédia, chaque apprenant disposant d'un poste de travail et d'un branchement Internet peut, avec un scénario pédagogique médiatisé en main, combiner judicieusement, de façon semi-autonome, la multicanalité d'Internet (son, image, écrit, etc.) et l'apprentissage du FLS. Cette «bonification pédagogique » d'Internet rejoint une préoccupation de premier ordre déjà exprimée par des chercheurs en LS et en langue étrangère (de Serres, 2004a; Furstenberg, 1997; Germain et Netten, 2004; Isabelle, 2002; Lancien, 2005; Lemeunier-Quéré, 2003) et signalée par d'autres en langue première, dont Lebrun (2005) et Tardif (2000).

\section{Pédagogie différenciée et compétences transversales ${ }^{8}$}

Le scénario pédagogique médiatisé permet à chacun de travailler et d'apprendre à son rythme sans par ailleurs contraindre quiconque à un travail de reclus comme cétait jadis le cas dans les laboratoires de langues munis d'isoloirs. Par les activités qu'il renferme, il suscite des contacts interpersonnels. Ces contacts peuvent se traduire par des échanges entre les apprenants mêmes, entre le pédagogue et les apprenants, voire entre les apprenants et la communauté. C'est là une nécessité signalée par des défenseurs d'un modèle socioconstructiviste de l'apprentissage d'une langue (Caws, 2005; Furstenberg, 1997; Germain et Netten, 2004; Kramsch et Andersen, 1999). Cette dimension entre en jeu dans la mesure où des activités d'un scénario donné, par exemple, se prolongent hors du cadre scolaire. En didactique des langues, la préoccupation d' "entrelacer » la langue et la culture est omniprésente (Chambers, 1999; Furstenberg, 1997; Kramsch et Andersen, 1999; Lemeunier-Quéré, 2003). Il importe d'allier sciemment la langue, le milieu, la culture et, bien sûr, le multimédia, en vue de développer des savoir-faire outrepassant les « savoirs bruts " (Germain et Netten, 2004, p. 10). 
Prenons le cas de la grille sondage évoquée plus tôt comme amorce (voir supra l'activité A.1). Cette grille pourrait, post scénario, s'inscrire dans un prolongement qui consisterait en un sondage effectué dans le milieu. Plus quanodine en langue seconde, une émulation semblable hors de la salle de classe est souhaitable. Elle génère un degré de motivation élevé chez l'apprenant et ce n'est là qu'un exemple parmi d'autres pour illustrer notre préoccupation réitérée quant à une implication authentique et signifiante de l'apprenant dans les tâches suggérées. De plus, notre choix d'accorder une attention toute spéciale aux interactions orale et écrite entre les pairs, qui se traduit par des tâches requises tantôt en dyade, tantôt en sous-groupe, tantôt en plénière, non seulement induit un climat de travail invitant, mais favorise de surcroît une participation active. Il se développe une coopération entre les apprenants où, conformément aux consignes du scénario, tous s'adonnent à la manipulation de l'information sous de multiples angles. La troisième séance de travail où s'effectue la préparation du reportage fournit en ce sens une illustration convaincante (voir infra l'activité C.6).

\section{C.6 La préparation du reportage}

Durée : \pm 45 minutes

1. En triade, préparez le synopsis du reportage

2. Répartissez les rôles :

a. Qui agira à titre de présentateur?

b. Qui agira à titre de reporter?

c. Qui agira à titre d'invité?

3. Si vous décidez de préparer une entrevue avec un invité, voyez http://www.bourgoing.com/formation/radio/09psychologie1.htm

4. Dans le menu de gauche, lisez les pages présentées en plaçant un $X$ sur la ligne lorsque vous avez terminé la lecture d'un sujet (p. ex. : X)

a. un peu de psychologie

b. I'utilisation prévue

c. les questions ouvertes et les questions fermées

5. Pour passer d'une page à l'autre, cliquez sur Page suivante

6. Informez-vous sur l'écriture radiophonique à http://www.bourgoing. com/formation/radio/21comprendre1.htm

7. Placez un $X$ sur la ligne lorsque vous avez terminé la lecture d'un sujet (p. ex. : X)

a. comprendre son sujet

b. le style parlé

c. la radio visuelle

8. Si vous avez prévu intégrer des sons à votre reportage, visitez http://www.bourgoing.com/formation/radio/18illustration.htm

9. Placez un $X$ sur la ligne lorsque vous avez terminé la lecture d'un sujet (p. ex. : X)

a. I'illustration sonore

b. types de sons

c. écrire avec les sons

10. Pour passer d'une page à l'autre, cliquez sur Page suivante

11. Préparez votre présentation selon les consignes de votre médiateur : présentation en direct ou à l'aide d'un préenregistrement audio.
Durée : \pm 15 minutes

1. Rendez-vous à http://www.rcinet.ca

2. Sélectionnez le français

3. Dans le menu, choisissez Émissions

4. Explorez les nouvelles!

5. Cliquez sur la date pour écouter la plus récente émission dans le format de votre choix ou sur l'icône Site Web pour accéder à la page de présentation de l'émission

6. Explorez également le http://www.tv5.org/TV5Site/info/actualite internationale.php

7. Voyez en images l'actualité mondiale, panafricaine, économique, etc. présentée par des professionnels.

Tout lecteur averti aura d'ores et déjà saisi qu'au cœur du modèle de scénarios pédagogiques médiatisés préconisés, la langue, le français en l'occurrence, supportera, voire propulsera l'apprentissage plus qu'elle ne le dominera. En d'autres mots, les composantes de la langue même ne font pas l'objet d'un enseignement direct et constant, de premier plan (p. ex.: la concordance des temps de verbe, le pronom complément d'objet, etc.). La préoccupation première repose plutôt sur la tâche qui commande la maitrise d'un contenu, lequel en retour met en valeur la langue apprise. À la différence de l'apprenant de français langue première, l'apprenant de FLS est plus ou moins en mesure, par exemple, de se fier à des repères de l'oral (p. ex.: pour le genre des noms) sur lesquels se base parfois le locuteur natif pour préparer une rédaction ou autres. Pensons aussi aux « habitudes culturelles", lesquelles sont intériorisées chez le locuteur natif, mais ignorées ou moins connues de celui qui évolue dans un contexte de LS. On pourrait ainsi multiplier les exemples pour contraster ces deux cadres d'apprentissage du français.

En dépit de tous les côtés positifs du scénario pédagogique médiatisé, on ne peut toutefois ignorer un second défi à surmonter:l'anxiété envers les technologies (de Serres et Lafontaine, 2005).

\section{Inquiétudes amenuisées}

Dans le souci de créer un milieu favorable à l'apprentissage pour tous, professeur et apprenants, il importe de ne pas occulter un facteur qui « donne souvent lieu à des sentiments d'anxiété [...] et d'insécurité [...] 》 (Isabelle, 2002, p. 86) : le recours à Internet. $\mathrm{Nul} \mathrm{n'ignore} \mathrm{que} \mathrm{la} \mathrm{technologie} \mathrm{génère} \mathrm{encore} \mathrm{chez} \mathrm{plusieurs}$ des craintes, des peurs: "peurs de ne pas pouvoir maitriser les problèmes techniques qui peuvent surgir (ordinateur en panne, pages qui ne s'affichent pas, sites introuvables, etc.) " (Barrière, 2006, p.3); peurs face au «changement [...] synonyme d'inconnu [qui engendre] l'effet négatif de l'anxiété des enseignants sur 
l'utilisation pédagogique des TIC $^{9}$ " (Isabelle, 2002, p. 86). Ce sont là des inquiétudes omniprésentes et légitimes dans le travail sur le terrain, mais comment les amenuiser?

Il existe plusieurs possibilités, l'une d'entre elles consiste à disposer dans le scénario même des «sites similaires pouvant être intégrés dans l'activité " (Barrière, 2006, p.3). En d'autres mots, il s'avère possible d'adjoindre aux activités principales certaines autres dites supplémentaires. Appelons-les « variantes ». Inscrite dans le thème choisi, la variante autorisera celui qui le désire, en guise de travail supplémentaire, par exemple, ou en raison de travers d'ordre informatique (p.ex.: un site fermé ou introuvable), à poursuivre son travail, toujours sur la thématique ciblée, mais d'une façon autre.

En guise d'illustration, nous présentons trois variantes (voir infra, la variante 1 - L'importance de la radio; la variante 2 - La radio dans la francophonie; la variante 3 - Le prix Judith-Jasmin). Chacune s'inscrit chronologiquement comme complément à chaque séance de 60 minutes qui précède la présentation même du reportage, soit l'activité D.8. En termes clairs, à la première séance de travail de 60 minutes qui comprend les activités A.1 à A.4 succède la variante 1 ; à la $2^{e}$ séance qui compte l'activité B. 5 s'ajoute la variante 2; à la fin de la $3^{\text {e }}$ séance composée des activités C.6 et C.7 s'inscrit la variante 3. Il va sans dire que le fait de disposer dans le scénario même d'un "surplus de matériel » en lien direct avec la thématique exploitée permet d'écarter ou à tout le moins de diminuer l'angoisse du professeur et de l'apprenant en ce qui touche les ratés courants de tout travail effectué à partir d'Internet.

\section{Variante 1}

L'importance de la radio

Durée : \pm 15 minutes

1. Allez à http://archives.radio-canada.ca

2. Choisissez Désastres et tragédies

3. Cliquez sur Le grand verglas de 1998

4. Sélectionnez De l'importance de la radio

5. Prenez connaissance des questions infra

6. Dites si les affirmations suivantes sont vraies ou fausses

7. Corrigez celles qui sont fausses

a. La journaliste Clothilde Seille n'a pas entendu d'autres témoignages que celui de la dame âgée. Faux. Clothilde Seille a entendu de nombreux témoignages semblables à celui de la dame âgée.

b. Parmi les numéros de téléphone donnés par la journaliste Clothilde Seille, on a entendu celui du Centre de psychologie Gouin.

c. La psychologue citée par Clothilde Seille conseille aux gens de rester isolés.

d. L'animateur suggère à la dame de demeurer chez elle pour s'occuper de sa maison et de celle de sa sœur plus âgée. e. Un habitant de Saint-Alexandre-d'Iberville se propose d'aider la dame âgée.

8. Lisez le contexte

9. Lisez Le saviez-vous?

10. Écoutez l'extrait audio en cliquant sur le bouton Écouter $( \pm 11$ minutes)

11. Répondez individuellement aux questions supra

12. À l'oral, comparez vos réponses à celles d'un partenaire.

\begin{tabular}{l} 
Variante $2 \quad$ La radio dans la francophonie \\
\hline Durée : \pm 10 minutes \\
1. Rendez-vous à http://www.tv5.org \\
2. En bas, à droite, cliquez sur WEBTV5 \\
3. Dans l'encadré blanc, tapez radio \\
4. Cliquez sur OK \\
5. Si désiré, voyez les vidéoclips suivants : \\
a. Africains francophones face à l'actualité autrichienne (les) $( \pm 3$ minutes) \\
b. Radios libres encore toutes proches (les) $( \pm 3$ minutes)
\end{tabular}

6. Prenez des notes. Indiquez ce que vous avez appris.

Variante $3 \quad$ Le prix Judith-Jasmin

Durée : \pm 10 minutes

1. Allez à http://archives.radio-canada.ca

2. Choisissez Vie et société

3. Cliquez sur Judith Jasmin, femme et journaliste

4. Découvrez le travail de cette journaliste :

a. écoutez l'extrait audio Femme de radio ( \pm 4 minutes)

b. écoutez l'extrait audio New York à Pâques ( \pm 3 minutes)

5. Sous Hyperliens, au bas de la page, cliquez sur Prix Judith-Jasmin.

Le troisième et dernier défi abordé ici repose sur un argument psychologique solidement ancré dans la gent enseignante (Barrière, 2006), inhérent à un enseignement-apprentissage, notamment à partir du scénario pédagogique médiatisé: le nouveau rôle du professeur.

\section{Métamorphose du rôle du professeur}

D'aucuns affirment que l'on sait encore peu de choses sur le nouveau rôle du professeur dans les situations d'interaction où se profile la technologie (Bressoux et Dessus, 2003); qu'un changement d'ordre pédagogique s'impose chez les professeurs (Barrette, 2005); que «l'enseignant n'est plus le référent en matière de langage ou de culture " (Barrière, 2006, p.3). Et cet état de faits prévaut dans le contexte de l'enseignement-apprentissage tant de la langue première que de la LS.

"Because the dynamics of interaction (and feedback-uptake relationships) in online environments differ from those in face-to-face interaction, teachers must be prepared for new ways of structuring 
tasks, establishing exchanges, guiding and monitoring interaction, and evaluating performance, not to mention mastering the relevant computer application "(Kern, 2006, p. 200-201).

L'outil pédagogique proposé dans cet article - le scénario pédagogique médiatisé - commande un changement de mentalités, « une rupture paradigmatique» (Tardif, 2000, p. 122), à savoir que:

«[...] le succès de la formation n'est plus fonction de la puissance des technologies, mais plutôt de la capacité des formateurs à exploiter leur potentiel afin de changer et d'améliorer l'efficience du processus d'apprentissage " (Sauvé, Wright et St-Pierre, 2004, p. 14).

Cette équipe de chercheurs rapporte une panoplie d'études témoignant de "la résistance au changement». Cette résistance, alimentée de méfiance et de fascination, est d'ailleurs abondamment documentée sur les plans tant théorique que pratique en ce qui touche le recours aux nouvelles technologies en langues première et seconde (Barrière, 2006; Eisenbeis et Marti, 2005; Fournier et Perreault, 2004; Isabelle, 2002; Lebrun, 2005).

Pourtant, l'outil proposé ici se jouxte simplement à la panoplie des moyens disponibles à ce jour dans l'enseignement-apprentissage d'une langue (de Serres, 2004a, 2006). Où donc blesse le bât? À la différence des autres moyens où le pédagogue occupe le premier plan dans la transmission des savoirs, celui-ci enjoint une décentration du «maître ». Décentration ne signifie toutefois pas rejet. Le pédagogue ne représente plus l'unique source du savoir, mais il ne faudrait pas s'y méprendre, le bon déroulement d'un scénario pédagogique médiatisé appelle un encadrement humain d'une grande compétence. Familiarisé avec le domaine de l'apprentissage des langues et le contenu du scénario exploité, en mesure de répondre aux interrogations des apprenants, sensible au maintien du rythme des activités, stimulant dans sa capacité à établir les liens entre le contenu du scénario et le contenu abordé en salle de classe, le médiateur agira comme figure de proue dans une exécution harmonieuse du travail. Il décidera si le retour sur une activité aura lieu en salle multimédia sous une forme orale entre pairs ou en classe, individuellement et par écrit, voire en devoir, donc hors de la classe. Il s'appliquera à «éveiller la curiosité des [apprenants]; [à] maintenir [leur] motivation; [à les] guider dans leurs démarches d'apprentissage; [à s'assurer qu'ils] intègrent, utilisent et réinvestissent les informations recueillies 》 (Fournier et Perreault, 2004, p. 94). Pour l'essentiel, «il devient un facilitateur d'apprentissage collaboratif » (Gevaert et De Pessenier, 2000, p. 1), il occupe une place centrale qui lui permettra d' ' [...] orienter, supporter et réguler le processus » (Larose, Grenon et Pearson, 2004, p. 74), mais non de le contrôler!
Le travail avec le multimédia, les scénarios pédagogiques en l'occurrence, commande une flexibilité certaine du pédagogue. Il s'agit d'une ouverture vers une nouvelle réalité, l'acceptation d'évoluer dans un cadre de travail différent de la salle de classe traditionnelle où il ne suffit plus de se positionner à l'avant-plan comme un chef d'orchestre, mais bien de se placer dans l'action, comme membre du groupe de travail. Tout bien considéré, le médiateur revêt les traits d'un "provocateur de développement " (Tardif, 2000 , p. 59), «[...] un aiguillonneur [pour qui] provoquer [...] est plus important que conduire » (Collot, 2002, p. 174). Le scénario pédagogique médiatisé offre "des tâches [qui] se révèlent être motivantes pour tous les niveaux dans la mesure où les apprenants utilisent de "vrais" documents, prennent conscience de leurs compétences et peuvent découvrir une utilisation réelle de la langue » (Barrière, 2006, p. 7). Alors pourquoi n'est-il pas plus répandu? Nous sommes tentée de répondre en formulant une hypothèse: se peut-il que le labeur requis dans sa préparation, l'angoisse éprouvée face aux technologies et le repositionnement du pédagogue soient particulièrement en cause?

\section{Conclusion}

Nous avons présenté trois défis reliés à une intégration réussie de ressources Internet dans l'enseignement-apprentissage du FLS chez l'apprenant universitaire à l'aide du scénario pédagogique médiatisé: 1. le temps nécessaire à la conception du matériel; 2. l'anxiété envers les technologies; 3 . le nouveau rôle du professeur.

Nous avons tenté de montrer quactuellement les ressources Internet peuvent, nonobstant leurs failles certaines, présenter une avancée dynamique dans l'apprentissage d'une langue seconde telle que le français. Le nouveau contexte d'apprentissage d'une langue suppose un recours à la technologie, plus précisément à Internet. Ce recours prend toute son importance dans la mesure où un travail de sélection judicieux de sites et de préparation d'activités prend forme. De là naît ce que nous avons appelé le "scénario pédagogique médiatisé ", outil au sein duquel l'apprenant occupe le centre de l'action. Quant au professeur, il dispense à titre de médiateur un encadrement humain exigeant et fort important. Les manipulations offertes dans un scénario se veulent porteuses de défis pour l'apprenant de FLS et d'implications pour le médiateur. Nous gardons en mémoire que la langue sert de moyen et non de finalité dans la réalisation du travail que commande le scénario. L'apprentissage devient dès lors optimisé. Pour l'essentiel, la cohérence des activités incite à apprendre; la diversité des manipulations de la langue suscite la motivation de l'apprenant; la bonification de la structure à l'aide de variantes contribue à réduire le degré de stress chez le médiateur et chez 
les apprenants. Voilà enfin un dispositif didactique qui intègre sciemment Internet en faveur de l'apprentissage du FLS.

Nous l'avons signalé dès les premiers paragraphes: en soi, les ressources d'Internet ne sont rien. Elles proposent « un rôle purement utilitaire ou fonctionnel du savoir [...] une automatique de l'instant [...]» (Lebrun, 2002, p.149). Par la trilogie heureuse qu'il incarne - technologie, pédagogie, apprentissage du FLS - et pour toutes les autres raisons évoquées dans ce texte, le scénario pédagogique médiatisé constitue à l'heure actuelle un outil pédagogique de choix dans l'enseignement-apprentissage du FLS. Son tout dépasse le simple recours à des sites Internet. Toutefois, sa conception exige sous divers angles un investissement important du pédagogue, investissement qui peut a priori et à raison sembler démesuré. Par contre, cet investissement atteste de l'apport incontournable des interactions générées à partir de contenus Internet mêmes. À la lumière de ce qui précède, il nous parait légitime d'avancer que le fait de relever les trois défis présentés (rappelons-les : le temps consenti à la conception du matériel selon une démarche thématique médiatisée et intégrée; l'anxiété envers les technologies; le nouveau rôle du professeur comme médiateur) tout en s'inscrivant dans une pédagogie active actualisée et actualisante convoque, peut-être contre toute attente cependant, une implication sans précédent du pédagogue et médiateur, ce défunt «maître de langues ».

\section{Remerciements}

L'auteure désire remercier les deux lecteurs anonymes de leurs commentaires judicieux, lesquels ont permis de bonifier le texte de cet article.

\section{Références}

Azurlingua (n.d.). Expressions idiomatiques. Récupéré le 25 septembre 2006 du site du cyber-magazine, http://www.bonjourdefrance.com/ index/indexexpresidiom.htm

Barrette, C. (2005, mars). Vers une métasynthèse des impacts des TIC sur l'apprentissage et l'enseignement dans les établissements du réseau collégial québécois. Mise en perspective. Clic: bulletin collégial des technologies de l'information et des communications, 57, 18-24. Récupéré le 3 avril 2006 du site du bulletin, http://www.clic.ntic.org/cgi-bin/ telecharger.pl?id $=57$

Barrière, I. (2006, 17 janvier). NTIC et FLE. Récupéré le 17 mars 2006 du site coopératif ÉduFLE, http://www.edufle.net/NTIC-et-FLE

Bressoux, P. et Dessus, P. (2003). Stratégies de l'enseignant en situation d'interaction. Dans M. Kail et M. Fayol (dir.), Les sciences cognitives et l'école (p. 213-257). Paris : Presses Universitaires de France.

Caws, C. (2005). Application de principes cognitivistes et constructivistes à l'enseignement de l'écrit assisté par ordinateur: perceptions des étudiants. Apprentissage des Langues et Systèmes d'Information et de Communication, 8, 147-166. Récupéré le 11 novembre 2005 du site de la revue, http://alsic.u-strasbg.fr/v08/caws/alsic v08 11-rec6.pdf

Chambers, G. N. (1999). Motivating language learners. Clevedon, Royaume-Uni : Multilingual Matters.

Collot, B. (2002). Une école de $3^{e}$ type ou « la pédagogie de la mouche». Paris: L'Harmattan.

Crystal, D. (2001). Language and the Internet. Cambridge, Royaume-Uni : Cambridge University Press.

de Serres, L. (2003). Sitographie commentée pour l'apprentissage du français langue seconde et langue première. Récupéré le 20 mars 2004 du site de l'École Internationale de Français de l'Université du Québec à TroisRivières, http://www.uqtr.ca/eif/sitographie/accueil.html

de Serres, L. (2004a). Le multimédia en classe de langue: un effet de mode? Québec français, 132, 62-65.

de Serres, L. (2004b). Stratégies de lecture communes d'hyperliens en langue seconde: étude exploratoire auprès d'universitaires francophones. Res Academica, 22(1), 79-102.

de Serres, L. (2004c, 14 avril). Tri sur le volet de 40 sites de FLE et repères pédagogiques. Récupéré le 3 mai 2004 du site coopératif ÉduFLE, http:// www.edufle.net/articlell1.html

de Serres, L. (2006). Voyager par Internet forme la jeunesse... en français! Réflexions: revue de l'Association canadienne des professeurs de langues secondes (ACPLS), vol. 25, $\mathrm{n}^{\circ}$ 3, 11-14.

de Serres, L. et Lafontaine, M. (2005). Utilisation des TIC : adaptation et validation de trois échelles de mesure de variables affectives. Revue canadienne des langues vivantes. Numéro spécial : les TIC dans l'enseignement-apprentissage des langues secondes, 62(1), 183-205.

Eisenbeis, M. et Marti, N. (2005). Créer un site pour mieux apprendre? Bilan d'expérience. Apprentissage des Langues et Systèmes d'Information et de Communication, 8 (numéro spécial Untele), 167-184. Récupéré le 3 avril 2006 du site de la revue, http://alsic.u-strasbg.fr/v08/eisenbeis/ alsic v08 17-pral.pdf

Exercices interactifs (n.d.). Récupéré le 4 octobre 2006 du site du Swarthmore College, section Humanities Division \& Interdisciplinary Programs - Français IB-2B, http://www.swarthmore.edu/Humanities/ gmoskos1/interexercice/inter2.htm

Fournier, H. et Perreault, F. (2004). L'enseignement stratégique et les TIC. Dans A. Presseau et M. Frenay (dir.), Le transfert des apprentissages: comprendre pour mieux intervenir (p. 86-101). Québec: Les Presses de l'Université Laval.

Furstenberg, G. (1997, juillet). Scénarios d'exploitation pédagogique. Le français dans le monde: recherches et applications, numéro Multimédia, réseaux et formation, 64-75. Paris : Clé International.

Germain, C.et Netten,J.(2004). Facteurs de développement de l'autonomie langagière en FLE/FLS. Apprentissage des Langues et Systèmes d'Information et de Communication, 7, 55-69. Récupéré le 3 avril 2006 du site de la revue, 
http://alsic.u-strasbg.fr/v07/germain/alsic v07 $\quad$ 08-rec2.pdf

Gevaert, R. et De Pessenier, J. (2000, 23 juin). Vade-mecum pour une didactique de l'Internet. Récupéré le 26 juillet 2005 du site de la Commission de l'Europe de l'Ouest de la Fédération Internationale des Professeurs de Français, section Textes et documents, http://www. ceo-fipf.org/article.php3?id article $=84$

Isabelle, C. (2002). Regard critique et pédagogique sur les technologies de l'information et de la communication. Montréal : Chenelière/McGraw-Hill.

Kern, R. (2006). Perspectives on technology in learning and teaching languages. Tesol Quarterly, 40(1), 183-210.

Kramsch, C. et Andersen, R. W. (1999). Teaching text and context through multimedia. Language Learning \& Technology, 2(2), 31-42. Récupéré le 27 septembre 2006 du site de la revue, http://lt.msu.edu/vol2num2/ pdf/articlel.pdf

Lancien, T. (2005, janvier-février). Les médias sur Internet. Le français dans le monde, 337, 23-26. Récupéré le 27 septembre 2006 du site de la revue, http://www.fdlm.org/fle/article/337/mediasinternet.php

Larose, F., Grenon, V. et Pearson, M. (2004). Les facteurs sociologiques et pédagogiques qui affectent les pratiques des enseignants du primaire au regard de l'informatique scolaire. Dans J. F. Desbiens, J. F. Cardin et D. Martin (dir.), Intégrer les TIC dans l'activité enseignante. Quelle formation? Quels savoirs? Quelle pédagogie? (p.59-80). Québec: Presses de l'Université Laval.

Lebeaupin, T. (1998). FLE-sitographie. Récupéré le 27 septembre 2006 de http://perso.orange.fr/fle-sitographie/internet.htm

Lebrun, M. (2002). Théories et méthodes pédagogiques pour enseigner et apprendre. Bruxelles : De Boeck Université.

Lebrun, M. (2005). eLearning pour enseigner et apprendre. Louvain-laNeuve, Belgique: Academia-Bruylant.

Lemeunier-Quéré, M. (2003, 15 octobre). Créer du matériel didactique: un enjeu et un contrat. Récupéré le 27 septembre 2006 du site coopératif ÉduFLE, http://www.edufle.net/Creer-du-materiel-didactique-un

Murano, M. (2004). La francophonie et la diffusion de la langue française. Apprentissage des Langues et Systèmes d'Information et de Communication, 7 , 191-201. Récupéré le 27 septembre 2006 du site de la revue, http://alsic. u-strasbg.fr/v07/murano/alsic v07 09-ficl.pdf

Perrenoud, P. (2004). Dix nouvelles compétences pour enseigner. Initiation au voyage (4 éd.). Paris : ESF.

Perrot, T. (n.d.). La palette. Récupéré le 27 septembre 2006 du site Peinture FLE, section Lexique, http://www.peinturefle.ovh.org/lexique/couleur.htm

Rézeau, J. (2001). Médiatisation et médiation pédagogique dans un environnement multimédia: le cas de l'apprentissage de l'anglais en histoire de l'art à l'université. Thèse de doctorat non publiée, Université Victor Segalen - Bordeaux 2, France. Récupéré le 27 septembre 2006 du site personnel de l'auteur, http://perso.orange.fr/joseph.rezeau/recherche/ thesePDF/acrobat.htm

Sauvé, L., Wright, A. et St-Pierre, C. (2004). Formation des formateurs en ligne: obstacles, rôles et compétences. Revue internationale des technologies en pédagogie universitaire, I(2),14-20. Récupéré le 27 septembre 2006 du site de la revue, http://www.profetic.org:16080/ revue/article $45 . h \mathrm{tml}$

Société Radio-Canada (2005). La télé l'a dit, donc c'est vrai. Pour les profs. Récupéré le $1^{\text {er }}$ mars 2006 du site de Radio-Canada, section Archives, http://archives.radio-canada.ca/294p.asp?IDCat $=10 \& I D D o s=323 \& \mathrm{~A}$ ctProf $=265 \& \mathrm{Nav}=\mathrm{AvPr} \& \mathrm{IDLan}=0$

Tardif, J. (2000). Intégrer les nouvelles technologies de l'information. Paris: ESF.

\section{Notes}

1 Les essais pilotes en salle multimédia facilitent notamment les ajustements nécessaires quant aux consignes et au niveau de compétence des apprenants ciblé a priori par le scénario : débutant, intermédiaire ou avancé.

2 Adapté du projet pédagogique "La télé l'a dit, donc c'est vrai » (Société Radio-Canada, 2005).

3 Dans les faits, l'apprenant ne tape aucune adresse http, il n'a qu'à cliquer dessus à partir d'un site compagnon conçu spécialement à son intention.

${ }^{4}$ Bien qu'a priori cette façon d'assurer le suivi en cochant les sujets lus puisse sembler infantilisante, elle offre en revanche l'avantage au médiateur de vérifier d'un simple coup d'œil, sans interrompre l'apprenant, le déroulement du travail de chacun accompli en salle multimédia.

${ }^{5}$ Les scénarios pédagogiques médiatisés peuvent offrir des contenus d'ordre soit purement linguistique (p.ex.: les pronoms relatifs) soit socioculturel (p. ex. : le reportage radiophonique). Dans ce texte, du fait qu'ils souscrivent à une préoccupation actuelle en enseignement-apprentissage du FLS, nous mettons l'accent uniquement sur les derniers. Quant aux premiers, bien qu'utiles, ils reposent sur une approche structuroglobale, jadis en vogue dans l'apprentissage des langues.

- Murano (2004) inclut dans une séquence pédagogique sur la francophonie trois types de savoirs dont celui de la linguistique et celui de la technique. Les premiers, d'ordre linguistique, renvoient notamment au fait d'acquérir du nouveau vocabulaire et de valoriser le réemploi de connaissances antérieures. Les seconds, dits «techniques", appellent entre autres l'utilisation d'Internet, du dictionnaire en ligne, du traitement de texte et d'une imprimante. Or c'est là, selon nous, une dimension utilitaire de base inhérente à un travail fonctionnel avec Internet. Lorsque nous signalons que le scénario pédagogique médiatisé ne requiert aucune compétence informatique particulière, nous référons au fait qu'il ne demande aucune compétence hors des connaissances de base que constituent le clic, la lecture et l'écoute de documents audiovisuels, c'est-à-dire des éléments avec lesquels tout apprenant nord-américain, parvenu à un niveau universitaire, s'est généralement familiarisé.

${ }^{7}$ Le corrigé s'applique dans ce cas-ci aux activités $\mathrm{A} 1$ et $\mathrm{A} 2$ de même quà la variante 1. L'expérience nous a enseigné qu'auprès d'étudiants universitaires d'origine asiatique, entre autres, le corrigé peut judicieusement 
éliminer maints questionnements et hésitations. L'apprenant s'y réfère soit comme rampe de lancement, soit pour raffermir sa compréhension de la consigne, donc pour se rassurer dans sa compréhension, et conséquemment, mieux poursuivre son travail.

${ }^{8}$ Ces préoccupations s'inscrivent dans un enseignement-apprentissage contemporain de la langue où l'on forme un citoyen du monde (Lebrun, 2002), où chacun est respecté dans ses différences, où chacun met en œuvre sa pensée créatrice, exploite la multicanalité des techologies, se donne des méthodes de travail efficaces, tire parti de l'information source et communique de manière appropriée.

9 Aux fins de cet article, Internet s'inscrit dans le terme générique TIC (technologies de l'information et de la communication) comme une technologie parmi tous les possibles. 